\title{
Unusual distal tibia BCOR sarcoma: A case report and review of imaging features
}

\author{
Puja Bharucha1, Nicole Harvilla², Rachel White², John Papadimitriou², Vincent $\mathrm{Ng}^{3}$, Michael Mulligan ${ }^{1 *}$
}

\author{
${ }^{\star}$ Correspondence \\ Michael Mulligan \\ Department of Radiology and Nuclear \\ Medicine, University of Maryland \\ School of Medicine, 22 South Greene \\ Street Baltimore, MD 21201. \\ Email: mmulligan@umm.edu \\ Telephone: (+1) 410-328-1291 \\ ${ }^{1}$ Department of Radiology and Nuclear \\ Medicine \\ 2Department of Anatomic Pathology \\ ${ }^{3}$ Department of Orthopaedic Surgery \\ Received: Dec 28, 2018 \\ Accepted: Feb 7, 2019 \\ Published: Feb 11, 2019
}

\begin{abstract}
Ewing's sarcoma is a common pediatric sarcoma. There is a subset of tumors similar to Ewing's sarcoma in several aspects, but negative for all known translocations. The BCOR sarcoma is one example. There are limited descriptions of the radiologic imaging findings. Previous reports describe the radiologic features as "aggressive" and "similar" to Ewing's sarcoma. This implies a permeative lesion centered in the diaphysis. Our case was geographic with a well-defined border and sharp zone of transition
\end{abstract}

Keywords: Ewing's sarcoma, BCOR sarcoma, Imaging.

\section{Introduction}

Ewing's sarcoma is one of the most common primary round cell sarcomas in the pediatric population. The most common chromosomal abnormality indicative of Ewing's sarcoma is the $t(11 ; 22)$ (q24; 12$)$ translocation which results in the fusion of EWSR1 and FLI1 genes [1-4]. There is a small subset of tumors which are similar to Ewing's sarcoma in several clinical and morphological aspects, but negative for all of the known Ewing's sarcoma associated translocations. These tumors were originally classified as "Ewing-like sarcomas" and are now considered "undifferentiated round cell sarcomas" according to the 2013 World Health Organization (WHO) classification [5].

The BCOR sarcoma is one example in the spectrum of undifferentiated round cell sarcomas. It was initially described by Pierron et al. in 2012 and they found it to represent approximately $4 \%$ of their transcriptnegative bone sarcomas [6]. The BCOR sarcoma is considered a separate biologic entity from Ewing's sarcoma based on gene profiling, which may have certain implications on clinical presentation, treatment, and outcomes [1]. There are only limited descriptions of the radiologic imaging findings of the cases reported to date.

\section{Case report}

The patient is a 10-year-old Hispanic male with no significant past medical history who presented with right lower leg/ankle pain and swelling. Patient reported initial symptoms for approximately 3 months and experienced a progressive decline in ambulation including the inability to play soccer and increased pain which was worse with activity. Patient's right lower leg and ankle pain persisted despite treatment with over the counter pain control medications and the use of compression wrapping to reduce the swelling. Patient reported fevers at the time of the onset of pain but denied weight loss, night sweats, or bruising. Vital signs and laboratory studies were only notable for an elevated erythrocyte sedimentation rate (ESR).

Initial radiographs of the right lower leg and ankle done at the time of presentation (Fig. 1) 
demonstrated a large, well circumscribed, lytic lesion located along the lateral aspect of the metaphysis of the tibia with subtle extension across the physis into the adjacent epiphysis and associated loss of the posterolateral cortex.
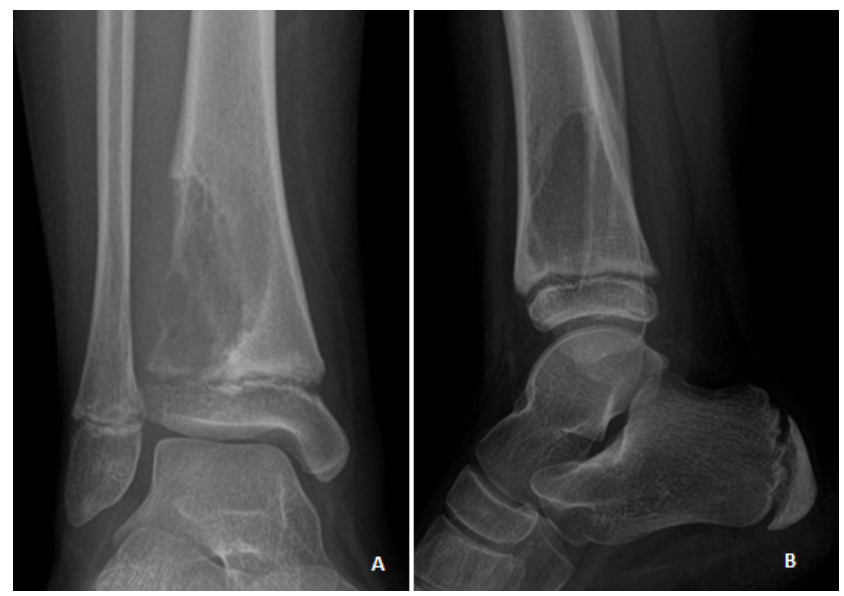

Fig. 1 Oblique (A) and lateral (B) radiographs demonstrate a large lytic lesion at the lateral aspect of distal tibia metaphysis involving the adjacent epiphysis, with destruction of the cortex.

There was no obvious periosteal reaction. The lesion measured approximately $5 \mathrm{~cm}$ superior-inferior by $2 \mathrm{~cm}$ anterior-posterior and $2 \mathrm{~cm}$ transversely. Initial differential diagnoses included aneurysmal bone cyst and juvenile giant cell tumor. Cross sectional imaging was subsequently performed with a noncontrast CT (Fig. 2) demonstrating similar features of a large lytic mass with focal absence of the posterolateral cortex but also demonstrating extraosseous extension and a $2 \mathrm{~mm}$ thick interrupted periosteal reaction along the posterior cortex.
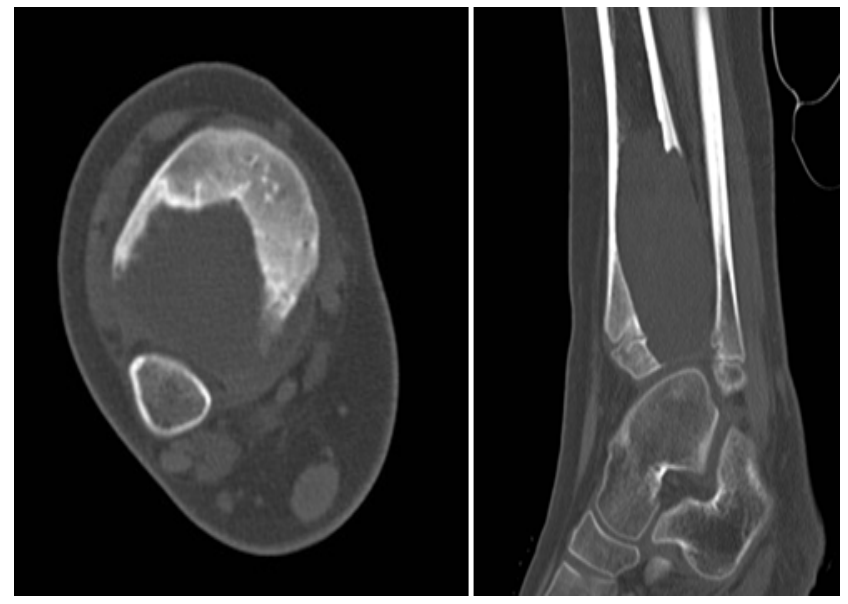

Fig. 2 Axial and sagittal noncontrast CT images demonstrates the large lytic mass in the distal tibia extending into the epiphysis with cortical bone loss and associated extra osseous extension of the mass.
Contrast enhanced MRI of the lower leg and ankle (Fig. 3) was notable for an indeterminate tumor centered in the distal tibial metaphysis extending through the physis and epiphysis with no definitive evidence of joint involvement. There was associated extraosseous extension measuring approximately $4 \mathrm{~cm}$ superior-inferior that had a well-defined pseudocapsule. No fluid-fluid levels were evident.

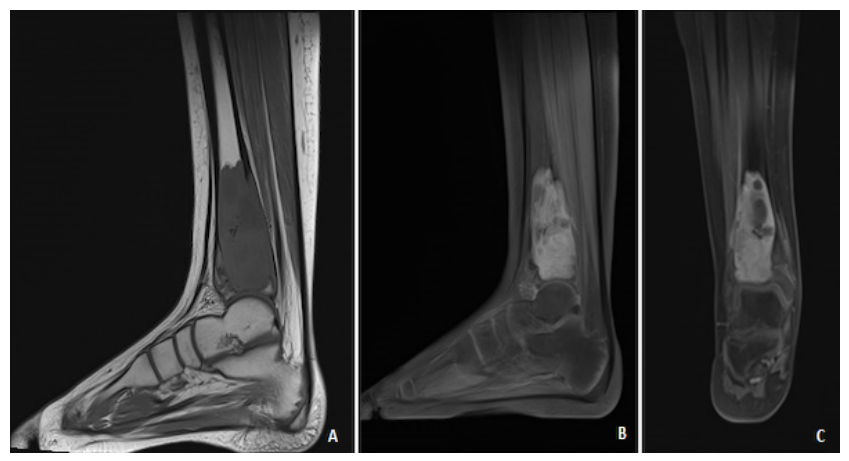

Fig. 3 Sagittal T1 (A) demonstrates the distal tibial metaphyseal mass with $\mathrm{T} 1$ intermediate signal similar to skeletal muscle measuring $5.7 \times 2.7 \times 2.9 \mathrm{~cm}(\mathrm{CC} \times \mathrm{AP} \times \mathrm{TV})$. The mass extends from the distal metaphysis through the physis and involves the epiphysis, with no definite evidence of extension into the joint. The mass extends posteriorly with cortical disruption. T1 fat saturated post contrast sagittal (B) and coronal (C) images demonstrate an enhancing mass with areas of central necrosis. Note that there are no fluid/fluid levels.

Patient subsequently underwent a CT guided biopsy of the lesion. Histologically a highly cellular tumor was noted that consisted of predominantly atypical ovoid vesicular nuclei, and scattered round to angulated nuclei, with an indistinct pale eosinophilic cytoplasm (Fig. 4A). There was focal nuclear pleomorphism and multifocal mitotic figures with atypical forms. The cells were arranged with no particular architectural pattern in a loose collagenous stroma. Immunohistochemistry was positive but limited for CD99, positive for vimentin and focally positive for EMA, SMA, and WT1. The PAS stain was strongly positive but PAS-D was negative. Immunohistochemical staining for BCOR was performed at an outside hospital and was strongly positive (Fig. 4B). Molecular sub-classification of the various BCOR genetic rearrangements was not performed. Immunohistochemical stains were negative for myogenin, CD31, desmin, pancytokeratin, and CAM 5.2. FISH and RT-PCR did not show any abnormality indicative of EWSR1-FLI1 or EWSR1-ERG gene rearrangement. 

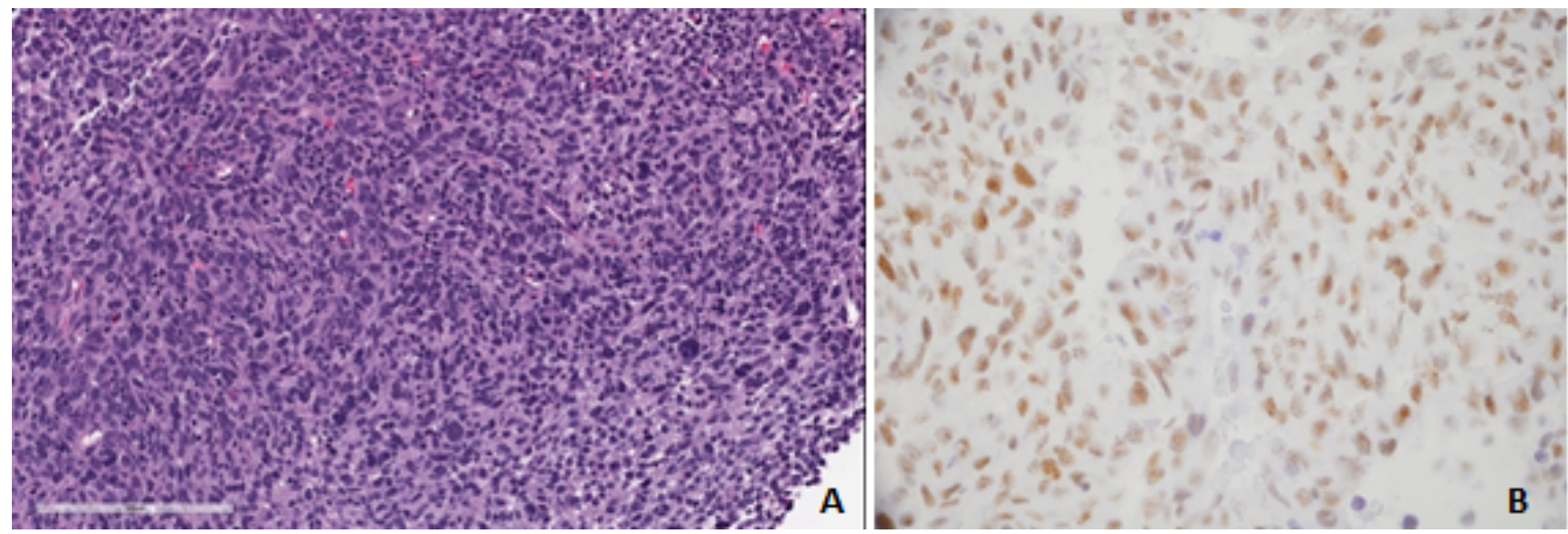

Fig. 4A,B. A - High power image from initial biopsy (hematoxylin and eosin stain, 40x) shows a highly cellular tumor composed of round to ovoid blue cells with marked size variation, atypical mitotic figures, and no distinct architectural pattern, arranged in a loose collagenous stroma. B - BCOR immunostain shows diffuse positivity in tumor cells.

Initial staging PET/CT demonstrated a highly FDG avid tumor with an SUV 14.1 but no evidence of metastasis. Neoadjuvant chemotherapy based on the Children's Oncology Group (COG) study AEWS 1031 protocol was administered with no significant response. Restaging radiographs and MRI demonstrated an increase in size and extent of the tumor with questionable invasion into the ankle joint. A below the knee amputation was ultimately recommended after tumor board discussion. The amputation was performed without postoperative complications and patient was discharged home.

Subsequent pathological evaluation of the below the knee amputation specimen of the right lower extremity (Fig. 5) demonstrated that the tumor measured $6.5 \times 3.4 \times 2.7 \mathrm{~cm}$ and was centered in the metaphysis of the distal tibia extending to the distal diaphysis, epiphysis, medullary cavity, and regional soft tissues between the distal tibia and fibula. No definitive involvement of the articular surfaces of the tibia or talus was identified, which was confirmed on histologic evaluation (Fig. 6). There was no evidence of lymph-vascular invasion. In comparison to the pre-treatment biopsy histologic appearance, the tumor was overall unchanged after neoadjuvant chemotherapy as shown on post-operative histology with more than $95 \%$ of tumor still viable (Fig. 7). Patient is currently 8 months post-surgery and is doing well with no evidence of disease. He is currently on week 17 of the Children's Oncology Group (COG) AEWS 1031 protocol, Regimen B.

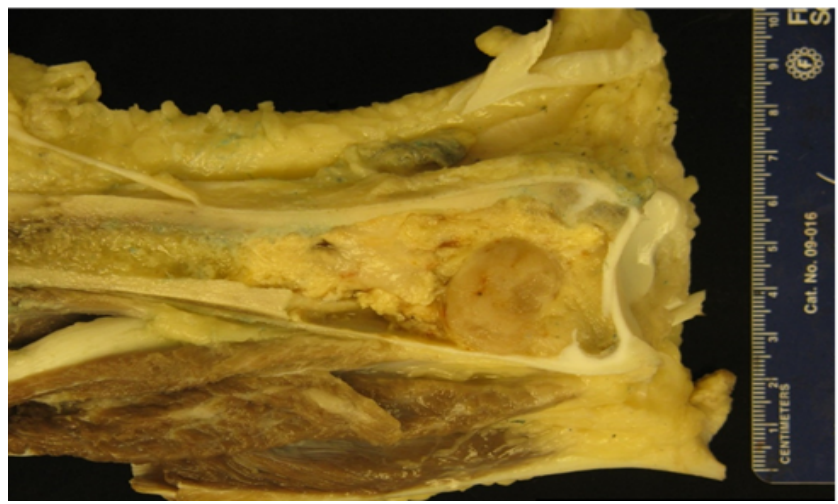

Fig. 5 Right below the knee amputation shows the distal end of the tibia marrow cavity completely replaced by the tumor which was displacing the overlying periosteum off of the bone. The lesion was characterized as soft with scattered necrotic material with cyst formation with a more discrete flesh-like pink region located in the metaphysis measuring $2.4 \times 2.0 \times 0.4 \mathrm{~cm}$.



Fig. 6 The resection specimen post-treatment (hematoxylin and eosin stain, 10x) shows the articular cartilage of distal tibia; articular surface at bottom right. Tumor has destroyed underlying bone, but does not invade into or through the articular cartilage. 


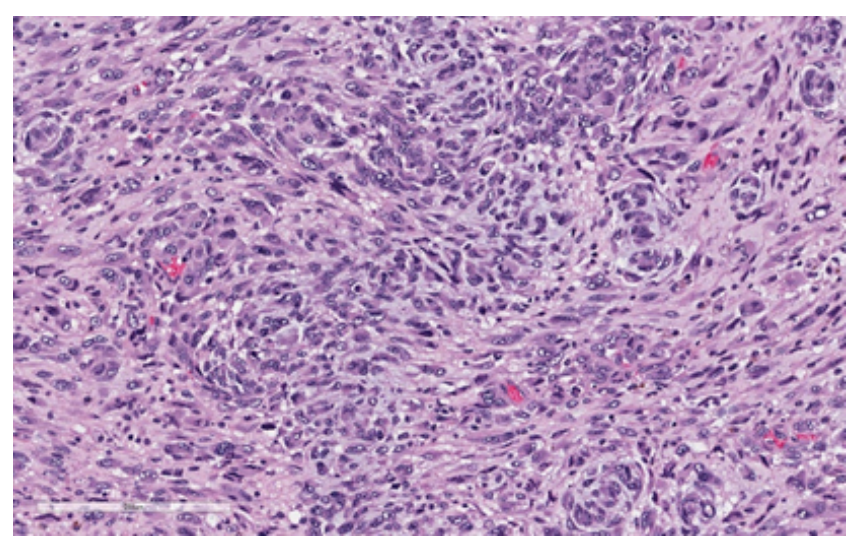

Fig. 7 The resection specimen post-treatment (hematoxylin and eosin stain, 40x) shows minimal decrease in cellularity, cellular atypia consistent with treatment effect, and atypical mitotic figures. No necrosis was present.

\section{Discussion}

The BCOR sarcoma is similar to Ewing's sarcoma in several clinical and morphological aspects. These include a predilection for long bones in a pediatric and young adult population with a shift towards a slightly older patient population relative to Ewing's sarcoma [1, 8]. One of the major differences in terms of demographics is a strong male predilection for BCOR sarcoma (M:F ratio of 3.4:1) compared to (M:F ratio of 1.5:1) Ewing's sarcoma $[\underline{z}, \underline{8}]$. Currently, the reason for the increased male predilection in relation to the paracentric inversion of the $\mathrm{X}$ chromosome is not well understood [9].

The majority of BCOR sarcomas that have been reported to date are bone-based tumors; however there have been many cases of discrete soft tissue based tumor $[\underline{1}, \underline{6}, \underline{7}, \underline{9}, \underline{10}]$. The first BCOR sarcomas were initially described by Pierron et al. who identified 24 BCOR-CCNB3 positive cases out of 594 sarcomas []․ 19 cases were bone-based with the pelvis $(\mathrm{N}=7)$ and femur $(\mathrm{N}=5)$ as the most common sites. There was 1 documented case involving the tibia. 5 of the cases originated in the soft tissues and were located in the neck, paravertebral, chest and abdominal wall, and pelvis. There was no description of the radiologic imaging findings.

Puls et al. subsequently analyzed and compared 10 cases of BCOR sarcomas with Ewing's sarcomas collected from 1998-2013 [9]. Similar to Pierron et al., the majority of the tumors were bone-based (7/10), 6 of which also demonstrated soft tissue extension. Three were soft tissue based. The locations in the order of the most common to least included the pelvis $(N=2)$, fibula $(N=2)$, femur $(N=1)$, tibia $(N=1)$, and calcaneus $(\mathrm{N}=1)$. The soft tissue tumors were located in the psoas, quadriceps, and gastrocnemius muscles. The radiologic and pathologic findings were illustrated only for 2 cases of tumors in the gastrocnemius and fibula. For the gastrocnemius tumor, a sagittal proton density image demonstrated a large hyperintense soft tissue mass. The fibula tumor was shown as a bright signal intensity, aggressive, destructive mass with a large circumferential soft tissue mass on a single coronal STIR MR image.

In 2014, Cohen-Gogo et al. performed a retrospective multicenter study which assessed the various presentation and outcomes of 26 patients with BCOR sarcomas [1]. The median age of the patients was 13.1 years with a male predominance, comparable to the studies performed by Pierron et al. and Puls et al. 21 cases were localized to the bone with the pelvis $(\mathrm{N}=8)$ and femur $(\mathrm{N}=6)$ as the most common sites, and 5 cases in the soft tissues. Similar to Pierron et al. and Puls et al., only 1 tibia case was identified. The imaging features of 12 bone tumors and 2 soft tissue tumors of the 26 cases included in the study were reported. All cases appeared as an aggressive lytic or mixed lytic and sclerotic lesion with cortical destruction and associated lobulated soft tissue mass without calcifications. CT imaging demonstrated the density of soft tissue involvement comparable to the surrounding musculature. On MRI, the lesions were primarily $\mathrm{T} 1$ hypointense and $\mathrm{T} 2$ hyperintense with heterogeneous, but intense enhancement. These CT and MR imaging features are similar to our current case.

Machado et al. in 2017 conducted a multicenter retrospective review of the morphological and immunohistochemistry profile of 41 undifferentiated round cell sarcomas which were negative for the pathognomonic EWSR1 gene rearrangement associated with Ewing's sarcoma [10]. 3 of the cases were identified as BCOR sarcomas. Detailed radiologic and pathologic findings were provided only for 1 of the BCOR sarcomas that involved a $22 \mathrm{~cm}$ long segment of the proximal femur. An axial fluid sensitive MR image showed a heterogeneous tumor located in the medullary cavity with associated cortical disruption and prominent surrounding soft tissue mass. 
Recently Kao et al. analyzed 36 molecularly confirmed cases of BCOR sarcomas [7]]. 20 cases were localized to the bone with the pelvis $(N=7)$, femur $(\mathrm{N}=5)$, and tibia $(\mathrm{N}=4)$ as the most common sites. 14 cases presented as discrete soft tissues masses localized in the trunk $(\mathrm{N}=7)$, extremities $(\mathrm{N}=4)$, and head and neck $(\mathrm{N}=3)$. Two cases were associated with visceral organ involvement such as the kidney, which had not been described previously. There was no description of the radiologic imaging findings.

Based on these studies, with 27 long bone cases (Table1), the usual imaging appearance of long bone
BCOR sarcoma has been similar to Ewing's sarcoma, that is a diaphyseal, permeative appearance with aggressive periosteal reaction and large surrounding soft tissue mass $[\underline{1}, \underline{6}, \underline{z}, \underline{9}, \underline{10}]$. Cohen-Gogo et al. and others have reported that the BCOR sarcoma can have imaging features of other primary bone tumors including; undifferentiated spindle cell sarcoma, and small cell osteosarcoma [1, 7]. These also would typically have a permeative lytic pattern with other aggressive features. Involvement of the tibia, as in our patient has been documented in a few cases, although not as one of the more common sites of involvement.

Table 1: BCOR Sarcoma Overview

\begin{tabular}{|c|c|c|c|c|c|c|}
\hline Authors & $\begin{array}{l}\text { Total } \\
\text { Number } \\
\text { of Cases }\end{array}$ & $\begin{array}{l}\text { Median } \\
\text { Age }\end{array}$ & Males & $\begin{array}{l}\text { Soft } \\
\text { Tissue } \\
\text { Cases }\end{array}$ & Long Bones & Imaging Findings Reported \\
\hline Pierron et al. & 24 & 14.3 & 16 & 5 & $\begin{array}{l}\text { Femur }(N=5) \\
\text { Tibia }(N=1)\end{array}$ & None \\
\hline Puls et al. & 10 & 15.5 & 9 & 3 & $\begin{array}{l}\text { Femur }(\mathrm{N}=1) \\
\text { Tibia }(\mathrm{N}=1) \\
\text { Fibula }(\mathrm{N}=2)\end{array}$ & $\begin{array}{l}\text { MRI image of an aggressive, destructive } \\
\text { mass with an associated circumferential } \\
\text { soft tissue mass. } \\
\text { MRI image of hyperintense soft tissue } \\
\text { mass in the gastrocnemius. }\end{array}$ \\
\hline $\begin{array}{l}\text { Cohen-Gogo et } \\
\text { al. }\end{array}$ & 26 & 13.1 & 17 & 5 & $\begin{array}{l}\text { Femur }(N=6) \\
\text { Tibia }(N=1)\end{array}$ & $\begin{array}{l}\text { Dedicated radiologic review of } 12 \text { bone } \\
\text { tumors and } 2 \text { soft tissue tumors. } \\
\text { All bone tumors presented as a poorly } \\
\text { marginated bone lesion with cortical } \\
\text { destruction and associated surrounding } \\
\text { noncalcified soft tissue mass. } \\
\text { MRI low to intermediate signal intensity on } \\
\text { T1 and high signal intensity of T2 with } \\
\text { heterogeneous, but intense enhancement. } \\
\text { Lytic }(N=7) \text { Mixed Lytic and Sclerotic }(N=4) \\
\text { Sclerotic }(N=1) \\
\text { Aggressive periosteal reaction in long } \\
\text { bone tumors }(N=4)\end{array}$ \\
\hline
\end{tabular}

\begin{tabular}{|c|c|c|c|c|c|c|}
\hline Machado et al. & 3 & $N / A$ & $N / A$ & $N / A$ & Femur $(\mathrm{N}=1)$ & $\begin{array}{l}\text { MRI image of heterogeneous tumor } \\
\text { located in the medullary cavity with } \\
\text { associated cortical disruption and } \\
\text { prominent soft tissue and muscle } \\
\text { invasion. }\end{array}$ \\
\hline Kao Y.C. et al. & 36 & 15 & 31 & 14 & $\begin{array}{l}\text { Femur }(N=5) \\
\text { Tibia }(N=4)\end{array}$ & None \\
\hline
\end{tabular}


Table 1 provides a summary of the location, median age, and radiological findings of the reported BCOR cases to date. Previous reports only generally describe the radiologic features of bone centered lesions as "aggressive" and "similar" to Ewing's sarcoma. This would imply a permeative type of lytic lesion centered in the diaphysis with aggressive periosteal reaction and a large surrounding soft tissue mass. Our case was centered in the distal tibia metaphysis and was geographic with a well-defined border and sharp zone of transition. It crossed the growth plate and involved the epiphysis. The initial radiographic appearance suggested a giant cell tumor or aneurysmal bone cyst. We present this case to add this less aggressive appearance to the imaging spectrum of these unusual bone sarcomas. BCOR cases centered in the soft tissues also have a nonspecific imaging appearance that is isointense to muscle on noncontrast $\mathrm{CT}$, with MR imaging showing decreased $\mathrm{T} 1$ and heterogeneous T2 signal. Contrast enhancement is variable.

Histologically, these are variably cellular tumors typically composed of monomorphic round to ovoid cells, with occasional spindled to angulated nuclei and finely dispersed chromatin arranged in a fascicular pattern, with a delicate vascular network [], 11]. The BCOR sarcomas can be differentiated from the Ewing's sarcoma family given the association with angulated nuclei, myxoid stroma, and spindle cells [4].

Given the rarity of these tumors, there is not an extensive understanding of treatment related effects and therapeutic responses are varied. There is increasing evidence which suggests that a majority of BCOR tumors are sensitive to Ewing's sarcoma based chemotherapy protocols []]. Our case showed no significant histologic response to standard chemotherapy. It is interesting to speculate if this could be related to the nonstandard imaging appearance. The patient remains disease free, at the time of this report, while continuing his chemotherapy regimen. Survival rates have been reported to be comparable to Ewing's sarcoma with a 5-year survival rate of $72-75 \%$ compared to $79 \%$ for Ewing's sarcoma [ $\underline{7}, \underline{9}]$. The incidence of metastatic disease has been reported to be between 11 and 40\% $[1, \underline{6}, \underline{2}]$.

\section{Conflict of interest}

The authors declare that they have no conflict of interest.

\section{Copyrights}

(c) Puja Bharucha et al, 2019; licensee OA Journal of Case Reports. This is an Open Access article distributed under the terms of the Creative Commons Attribution License (http:// creativecommons.org/licenses/by/4.0), which permits unrestricted use, distribution, and reproduction in any medium, provided the original work is properly credited.

\section{References}

1. Cohen-Gogo S, Cellier C, Coindre J, et al. (2014) Ewing-like sarcomas with BCOR-CCNB3 fusion transcript: A clinical, radiological and pathological retrospective study from the Société Française des Cancers de L'Enfant. Pediatr Blood Cancer. 61: 2191-2198.

2. Delattre O, Zucman J, Melot T, et al. (1994) The Ewing Family of Tumors -- A Subgroup of Small-Round-Cell Tumors Defined by Specific Chimeric Transcripts. N Engl J Med. 331: 294-299.

3. Delattre O, Zucman J, Plougastel B, et al. (1992) Gene fusion with an ETS DNA-binding domain caused by chromosome translocation in human tumours. Nature. 359: 162.

4. Kilpatrick SE, Reith JD, Rubin B. (2018) Ewing Sarcoma and the History of Similar and Possibly Related Small Round Cell Tumors: From Whence Have We Come and Where are We Going? Adv Anat Pathol. 25: 314-326.

5. Fletcher C, Chibon F, Mertens F. (2013) Undifferentiated/ unclassified sarcomas. In: Fletcher C, Bridge J, Hogendoorn P, et al, eds. WHO Classification of Tumours of Soft Tissue and Bone. IARC Press, Lyon, France.

6. Pierron G, Tirode F, Lucchesi C, et al. (2012) A new subtype of bone sarcoma defined by BCOR-CCNB3 gene fusion. Nat Genet. 44: 461-466.

7. Kao Y-C, Owosho AA, Sung Y-S, et al. (2018) BCOR-CCNB3 Fusion Positive Sarcomas: A Clinicopathologic and Molecular Analysis of 36 Cases With Comparison to Morphologic Spectrum and Clinical Behavior of Other Round Cell Sarcomas. Am J Surg Pathol. 42: 604-615.

8. Peters TL, Kumar V, Polikepahad S, et al. (2015) BCOR-CCNB3 fusions are frequent in undifferentiated sarcomas of male children. Mod Pathol. 28: 575-586.

9. Puls F, Niblett A, Marland G, et al. (2014) BCOR-CCNB3 (Ewinglike) Sarcoma: A Clinicopathologic Analysis of 10 Cases, In Comparison With Conventional Ewing Sarcoma. Am J Surg Pathol. 38: 1307-1318.

10. Machado I, Yoshida A, Morales MGN, et al. (2018) Review with novel markers facilitates precise categorization of 41 cases of diagnostically challenging, "undifferentiated small round cell tumors". A clinicopathologic, immunophenotypic and molecular analysis. Ann Diagn Pathol. 34: 1-12.

11. Schaefer IM, Fletcher CDM (2018) Recent advances in the diagnosis of soft tissue tumours. Pathology. 50: 37-48. 